\title{
Impact of functional/cognitive and emotional advertisements on image and repurchase intention
}

\begin{abstract}
In this study, we attempted to explore the role of chain steakhouse image (functional, symbolic, and experiential), pleasure, arousal, and brand satisfaction in building customer repurchase intention and to uncover the moderating impact of functional/cognitive and emotional advertisements (ads). A field survey was conducted for data collection. A quantitative approach was employed to analyze the data. Findings from the structural analysis showed that image, emotions, and satisfaction played a significant role in generating intention, both pleasure and satisfaction acted as mediators, and hypothesized associations were mostly supported. Moreover, the relationships among functional image, pleasure, and brand satisfaction within the proposed theoretical framework were significantly moderated by functional/cognitive and emotional ads. Implications for researchers and practitioners are discussed. Current research provides chain steakhouse management a better understanding of the underlying complicated mechanism of customers' repurchase decision generation process.
\end{abstract}

Keyword: Chain steakhouse image; Functional/cognitive ads; Emotional ads; Brand satisfaction; Repurchase intention 